\title{
Environmental Management of Production Processes in Heating Systems when Receiving Magnetic Water in Reagent- Free Method with the Aim of Environmentalization
}

\author{
Petro Kulikov ${ }^{1}$, Olena Bondar ${ }^{2}$, Nataliia Zhuravska ${ }^{3 *}$ \\ ${ }^{I}$ Kyiv National University Of Construction And Architecture, Ukraine \\ ${ }^{2}$ Kyiv National University Of Construction And Architecture, Ukraine \\ ${ }^{3}$ Kyiv National University Of Construction And Architecture, Ukraine \\ *Corresponding Author E-Mail: Nzhur@Ua.Fm
}

\begin{abstract}
It has been proved that the environmental management of industrial systems of a heat supply, under the terms of the application of the method of preparation of reagent-free industrial water depends on ensuring the implementation of its basic principles. It is shown that the electromagnetic interaction of multi factorial criterial components of the process of magnetization of water is performed through the mechanism of feedback in the heating system. And the mechanism of feedback in the heating system is characterized by the properties of loop negative feedback and therefore is auto regulation of the process of magnetization of water takes place. Quantitative indicators of integral system of control over the process of magnetization of water in heating systems were defined. Both the graduation orderliness of mass exchange process and its additive function must be within $\leq 1$.
\end{abstract}

Keywords: environmental management, greening, reagent-free water treatment

\section{Introduction}

One of the internationally recognized instruments to reduce the impact on the environment is environmental management [1-3]. According to the ISO 14000 environmental aspect being one of the basic principles of industrial facilities, interacts with the environment and determines the structural components of the change in its condition (atmosphere) at the local-territorial level. This paper emphasizes two areas of environmental impact: negative, in potential, possible pollution of the atmosphere; positive - changes associated with human activities, that are characterized by resource-saving technologies. In this direction we carried out to our previous studies on the development of a reagentless method for the preparation of industrial water, in electromagnetic fields in heating systems [7].

Another international document ISO 14001-2004 presents recommendations of the environmental management system when the production activities of enterprises should not lead to negative anthropogenic impacts on the environment, through the development of specific conservation programs. The document notes that a key indicator of the environmental performance of enterprises are specific informative indicators of control, which display information regarding the actual prominent parameters of the process of magnetization of water and their specific indicators, which provides under the conditions of organized processes of mass transfer in the heating system (the process of magnetization of water). The consequence of this scientific hypothesis is the stability of the magnetic interactions in the heating system and confirmation of the possibility of reagentless treatment of water in electromagnetic fields in heating systems.
Indicators-recorders of the dynamics of changes of structuralfunctional properties (relations and interactions) of the process of magnetization of water are the driving forces in the environmental activities of an industrial facility. Such studies are relevant because they not only determine the reliability of the engineering plant for carrying out the magnetization of water but also allow to forecast for the future efficiency of the reagentless method of water treatment in the electromagnetic fields of heating systems. And finally, the creation of indicators of registration of potentially possible changes in the structural and functional properties of the material flows of the processes of magnetization of water, refer to the organization of systematic observations of the change of certain parameters for the change of certain parameters. Previously established conclusions regarding the formation of man-caused risks in the heating system stipulate potentially possible anthropogenic changes in the atmosphere due to steam emissions.

Reagentless method of water treatment in electromagnetic fields in heating systems [4] relates to nanotechnology and, in addition, is determined by the small period of its existence. Due to the fact that there is still scientific applied issues about this process that one should pay attention to. First, that potentially possible risks (industrial safety) contribute to anthropogenic impact on the atmospheric air (industrial emissions) and, in the case of critical situations (e.g. power failure on a local or territorial level) - a violation of the thermodynamic mass transfer dynamics of material flow in heating systems for energy-thermal facilities construction industry, as a consequence of local thermal pollution of the atmosphere [2]. Thus, there is a need to establish the cause of changes in relations between components of the technological process, affecting the interests of such industries: environment, 
energy, economy. The uniqueness of the water treatment with electromagnetic fields in the heating systems, the lack of scientific and technical solutions definition, if necessary, control mechanisms for management of technogenic-ecological security (support of the dynamic equilibrium between intentions and their implementation in production processes and the elimination of negative impacts on the atmosphere). And management solutions to production processes (environmental management) is the basis prerequisite of resource consumption, low-waste production technologies in heat supply systems, and the result of the implementation of magnetization processes in electromagnetic fields is environmental compatibility.

When developing the methodological basis of the environmental management system the following research areas are combined: a definition based on predetermined parameters of the process of magnetization of water specific indices and their correlation coefficients (oscillation frequency of electromagnetic field, causes of magnetic field induction); determination of anthropogenic allowable levels (max, min, opt) considering specific correlation coefficients; development of interpretation methods of obtaining results; development of methods of forecasting and mathematical modeling.

On the basis of the obtained results the correctness of the approach assessment of the process of magnetization of water should be noted, in our opinion, when the components of integrated systems of indicators should be limited, but fully reflect the structural and functional state of material flow in the heating system. Under dynamic operating conditions, engineering installations of nonchemical water treatment in electromagnetic fields (monitoring of adsorption processes at the interface: solid surface (piping) material flow system of a heat supply. Therefore, in the future, for the development of integrated systems of indicators this principle should be accounted.

Due to the fact that the performance of the company's environmental management system depends on the use of specific indicators reflecting the nature of the activities of production processes, the topic of scientific publications is devoted to this issue.

\section{Results and their Discussion}

Energy systems of cities are the main consumers of electric and thermal energy. But in the consumption of energy there are losses and it is due to the fact of the low efficiency of the heat pipes in the distribution of hot water and steam for industrial and household structures in urban areas [11]. The author emphasizes that a certain role in energy savings can be obtained by conversion of city production. That is, such achievements are obtained by using reagent-free water treatment with electromagnetic fields in the heating systems [3-5]. Previous established scientific laws of obtaining magnetic water and possibilities of their practical use give possibility to state that energy-thermal processes of heating systems at presence of multi-component and multi-level components of the system (intention-production-society-nature) are related to such disciplines: energy, engineering, ecology, economy. Therefore, the principle of consistency is the leading principle at studying such multi component complex, which given the concept of mass transfer provides an approach to them.

Mass transfer processes in the heating system are associated with the transfer (of substance or mass of magnetic water) from one phase to another (solid - liquid). Diffusion mass transfer of components of the heating system [7] is characterized by the change in free energy in these components, individual parts of the system. As a result of these processes there is a change, specific of volume, free energy of a component of the parameter that best reflects the physical basis of this phenomenon. In addition, the use of the ideas of thermodynamics allows us to conclude that all spontaneous processes in the heating system go in one direction from an ordered state to disordered state.
Multifactorial state of the components of the process of magnetization of water and multilevel nature of the relationships between them causes (Fig. 1) the establishment of the main principles of management decisions on optimization of production activities. In the preparation of the water [4] in heating systems we deepened the knowledge about the fundamental interactions of material flows in the process of obtaining magnetic water under certain physical parameters. Thus, the electromagnetic interaction is due to electric and magnetic fields: electric field and its appearance is the presence of electric charges and magnetic field in their motion. The nature of electromagnetic interaction is determined both by positive and negative charges.

Physical state of material flow in the heating system is determined mainly by the intermolecular forces, which in its structure is the electromagnetic interaction which is consistent with the fundamental laws of electrostatics and electrodynamics: Coulomb's law, Ampere's law and others. But it's most general form is described in the electromagnetic theory of Maxwell, which is based on the fundamental equations which link together the electric and magnetic fields. Physical processes confirm the interaction, in our case, of separate components of the electromagnetic interaction parameters and their specific indicators of the overall additive component functions of the process of magnetization of water (table. 1).

To assess and compare the dynamics of changes of the process of magnetization of water in systems engineering, it is convenient to use specific indicators. As to the levels of acceptability of specific indicators it is necessary to add, that their function is growing monotonically to some value of its argument - order material flows magnetization of water, as well as for preliminary levels. Taking into account the specific technological parameters of magnetization and, given the hierarchical approaches of multifactor components (specific indicators - the index of correlation between technology flows), we have found the optimal limits of structural-functional state changes of the heating system, which should be at the level of $\leq 1$. The estimated level - graduation is a landmark to maintain reagent-free water treatment according to certain parameters and their specific indicators.

Analysis of the data in the table, their relativeness to the technological process, allow us to state that the maximum gradation of the technological process for the maximum and minimum parameters differ from optimal by the fact that the strengthening or weakening of action of electromagnetic fields in certain quantities loosen the interaction between specific indicators.

Establishment of the maximum gradations of the process of water magnetization became possible thanks to our systematic approach in conducting and evaluating nonchemical water treatment in electromagnetic fields.

However, the direction of the goal - the preservation of mass transfer order of material flows (table. 1) is carried out by electromagnetic interaction by getting the relevant information based on the feedback of this mechanism of feedback characterized by the properties of negative feedback loop, and therefore auto regulation of the processes of magnetization of water takes place continuously. That is, identifying positive and negative features (gradations) of preparation of technical water in electromagnetic fields in systems of a heat supply determines the feasibility of water treatment in the application of the appropriate method. A very important principle of environmental management, which is implemented in this technology is not only obtaining of expected material and energy feedbacks to enhance mass transfer processes, but also saving natural (water $10 \%$ ) and energy (33\%) resources.

Environmental management of production processes is directly connected with environmental and economic issues.

The ecological and biological components of a potential manmade hazard include biological formations, which are formed through reagent treatment of water in heating systems and in the transition to reagentless preparation of water in heat supply 
systems they are reduced due to the breakage of the biomass that affects material flows (precipitation) of magnetic water.

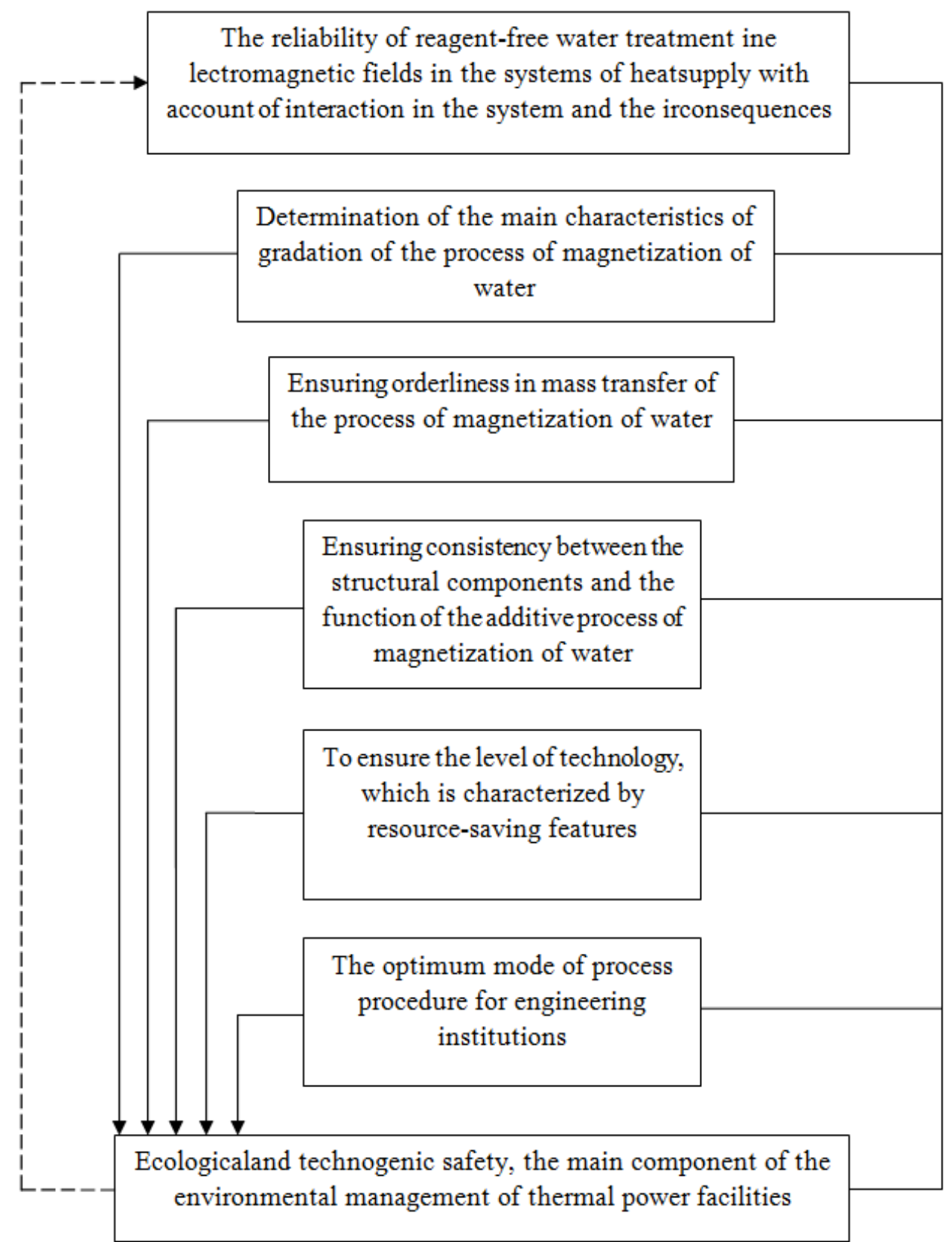

Fig. 1: Scheme of the electromagnetic interaction of the components of the mechanism of additive functions of the process of magnetization of water on autoregulatory opportunities feedback: direct connections, backward connections - - -

Table 1: Characteristic structural components, and functional changes in the dynamics of the process of magnetization of water according to the strength of the interaction of electromagnetic fields

\begin{tabular}{|c|c|c|c|c|c|}
\hline \multirow{2}{*}{$\begin{array}{l}\text { Specific indicators to determine the } \\
\text { parameters of magnetic water, such as } \\
\text { mathematical derivatives-gradation of the } \\
\text { efficiency of magnetization of water }\end{array}$} & \multicolumn{3}{|c|}{$\begin{array}{c}\text { The level of gradation of the dynamics of structural-syllabic } \\
\text { status of the heating system }\end{array}$} & \multicolumn{2}{|c|}{$\begin{array}{l}\text { An additive function of a reagentless } \\
\text { water treatment in heating systems }\end{array}$} \\
\hline & $\begin{array}{l}\text { the maximum } \\
\text { acceptable level }\end{array}$ & $\begin{array}{l}\text { the minimum } \\
\text { acceptable level }\end{array}$ & the optimal level & $\begin{array}{l}\text { the formation of } \\
\text { the technical risks }\end{array}$ & $\begin{array}{l}\text { effectiveness of } \\
\text { water treatment }\end{array}$ \\
\hline $\begin{array}{l}\text { The frequency of oscillations of the } \\
\text { electromagnetic field }\end{array}$ & 0,8 & 0,01 & 0,45 & \multirow{2}{*}{0,54} & \multirow{2}{*}{0,22} \\
\hline $\begin{array}{l}\text { The magnitude of induction of the } \\
\text { electromagnetic field }\end{array}$ & 0,96 & 0,05 & 0,56 & & \\
\hline
\end{tabular}

For clarity, the concept of the mechanism of structural and functional relationships of the specified system of a heat supply, contributing to the performance of reagent-free water treatment (RWT) in the electromagnetic fields is achieved by energyefficient heating system with higher level of environmental safety. The dynamics of changes in the technology of water treatment was previously investigated on a laboratory stand over a long term period to be subsequently in the heating system $[4,5]$.
At structural and functional interaction between components of the RWT for the first time we established structural and unifying role of ecological and anthropogenic factors (the relation).

Building of such a program-scheme for further research has become a necessity for the disclosure of the concept of sustainability of the proposed system of RWT in electromagnetic fields. 
We have proposed a scientific hypothesis that the negative charge of the metal prevents further transfer of the metal ions in water solution and causes inter-ion interaction of transition of cations in a metal, which is the barrier for the formation of monomolecular electrolyte system with positively charged ions. That is, the technological efficiency of the proposed heating system is not possible without regulation of ecological and technogenic situations in the application of the RWT due to the fact that undesirable structural and functional interactions disrupt these processes [3].

The basis for the development of the program were the following ecological and technological approaches:

- the principle of system approach which allows to describe structural and functional relationship between the components of the method of RWT;

- the principle of optimization of technology of the RWT due to the coherence of the scientific mechanism of physical water treatment with the relevant laws of general ecology;

- the principle of conservation of self-regulation of heating systems (technical and functional reliability of the RWT);

- the principle of maintaining a balanced flow of processes in the system "man-made impacts - the result of the influence";

- the effectiveness principle of RWT in electromagnetic fields;

- the principle potentially environmental risks of the RWT technology (prospect).

Analysis of the structural components of the programmes of the scheme shows that they are fully responsible for tactical scientific principles in relation to the strategic direction of obtain a highly efficient, environmentally friendly technologies of the RWT in electromagnetic fields.

The water treatment technology was carried out in the following sequence:

1) tap water was treated with 2-chamber device with paralle electrodes in a constant electric current field under the condition of changes in automatic mode of the signs of the electrodes in the chambers and the direction of water in them;

2) due to the potential difference of electric current between the electrodes (about 2.5-3V) water softening and partial coagulation of certain elements in it occur due to electrolysis;

3 ) purification of water from precipitation and softening to a $\mathrm{pH}$ of $\leq 6.0 \mathrm{~V}$ in the apparatus for water magnetizing in the highfrequency electromagnetic field with isolated electrodes at a frequency of $1 \ldots X \mathrm{kHz}$

4) in the water between the electrodes a magnetic field is generated that leads to the destruction of cluster and inter-cluster hydrogen bonds and it turns into active monomolecular liquid with a dipole, positively charged ions;

5) later in container where RWT was sent in the electromagnetic field a certain number of biocidal additives are sent;

6) the RWT is fed to the disperser-mixer of rotating vibrating type, and then through pipelines dispersed and homogenized solution with magnetized water enters the system for use.

As to air pollution by emissions from the technological process, it should be noted, that when the ratio of heat of vaporization to the specific heat capacity of water is 0.9 , it is not a threat to air pollution in the area of energy-thermal object.

\section{Conclusion}

1. It has been established that the method of reagentless water treatment in electromagnetic fields is determined by multyfactorial criteria components of the process of magnetization of water in heating systems.

2. It is shown that the electromagnetic interaction of the technologies of magnetization of water is through the mechanism of feedback. This mechanism is characterized by the properties of the circuit with negative feedbacks and therefore, there is a constant autoregulation of the process of magnetization of water.

3. Quantitative indicators of integral system of control over the process of magnetization of water were defined; both the
The proposed ecological-economic model of the process of magnetization of water, from a production point of view, is hierarchically organized on the basis of adequate forms of control orientation of technological processes, is performed by the integral system of indicators proposed and developed by us. Indicatorsrecorders of the process of magnetization of water are the driving force $s$ of environmental management on energy-thermal installation, which not only allows to set certain gradation of specific indices, but also to determine, based on certain parameters and their specific values, their optimum gradations.

Considering the issue of anthropogenic impacts from thermal power equipment, the use of reagent-free water treatment with electromagnetic fields, it can be stated that virtually there is no direct impact on the atmosphere on local-territorial level. One can only you attest to the presence of mediated effects, regarding the use of natural resources and energy, the nature of the change which is of positive nature, a significant level of safety.

One of the areas of environmental economics is the determination of costs and potential losses from introduction of this technology of water treatment in heating systems (analysis of capital expenditure and analysis of current costs; analysis of the impact of environmental activities on the formation and evaluation of financial results of activity of object of research).

In this scientific publication, we only covered a statement of the fact regarding resource saving (water, energy), orientation of reagentless water treatment in electromagnetic fields in heating systems. At this stage of economic assessment of natural resources one can state that it is the monetary expression of their economic value $[3,8,9]$

The modeling of the processes of magnetization of water is based on specific indicators and factors and criteria, which perform the function of correlation of the process of magnetization of water in heating systems.

Processing of industrial water in electromagnetic fields in heating systems is described by the system of equations (1):

$\left\{\begin{array}{l}\frac{d x_{f}}{d t}=F_{i}\left(P_{t} \cdot S\right) \\ \frac{d x_{\phi}}{d t}=\Phi_{i}\left(P_{t} \cdot C\right)\end{array}\right.$

where: $F_{i}\left(P_{t} S\right)$ is a function that characterizes the structural components of the changes in the system when using specific indicators at different levels of their graduation (ordering of the system of mass transfer);

$\Phi_{i}\left(P_{t} \cdot C\right)$ - functional changes in the heating system when using specific indices (mathematical derivatives from defined parameters of the process of magnetization of water in electromagnetic fields) at various levels of their gradation given additive function of the process of magnetization of water.

The calculations are carried out with the aim of identifying of potentially-possible man-made risks that may arise from independent from us reasons, which will affect the dynamics of the process of water magnetization within certain parameters and indicators.

gradation of order of mass transfer process, and its additive function. They must be within $\leq 1$.

\section{Acknowledgement}

The predetermined scientific regularities have been obtained through continuous collaboration with the scientific leader of the candidate's thesis E. Malkin, Sc.D, professor, National University of Construction and Architecture; the possibilities of their practical use and implementation, thanks to cooperation with $\mathrm{M}$. Ishchenko, Ph.D.

This allowed further developing and using the multilevel components of the system, which will be presented in the following publications. 


\section{References}

[1] Shevchuk VY \& Satalkin YM (2004), Environmental management. Textbook, Lybid, 432 pp.

[2] Udod VM, Kuznetsov VV \& Wolosky AS, Technoecology. Kyiv, (2007), $195 \mathrm{p}$

[3] Zhuravska NY (2015), Energy saving systems of a heat supply by treatment of water in electromagnetic fields . Dis. Of the Cand.tech. Sciences, $163 \mathrm{p}$.

[4] Malkin ES, Furtat IE, Zhuravska NY \& Kovalenko NA (2015), Water treatment system in electromagnetic fields. The patent for usefull model 100236.

[5] Malkin ES, Furtat IE \& Zhuravska NY (2015), The system of preparation of magnetized water in the electromagnetic fields of water and solutions and compounds based on it. The patent for useful model , 102494.

[6] Zhuravskaya NY (2015), Resource-saving materials, constructions, buildings and structures. Educational and scientific Institute of correspondence distance learning of the National University of water management and nature resources use Sciences. ZB, V. 30 19-28.

[7] Draganov BH, Engineering and application of heat in agriculture. Agropromizdat, (1990), $463 \mathrm{p}$

[8] Statiukha GO, Sokolov VA \& Abramov B (2010), On the matter of quantitative evaluation of environmental safety in the EIA. EasternEurope. Journal of advanced technologies 6, 44-46.

[9] Izmalkov VI \& Izmalkov VA (1998), Technogenic and ecological safety and risk management. The research center for russian academy of sciences, $482 \mathrm{p}$.

[10] Baran BA (1999), The influence of magnetic field on the micelle formation and coagulation of barium sulfate in water solutions. IFH, T. 73, 11, 2089-2090.

[11] Zlobin YV, Fundamentals of ecology. Kyiv, (1998), 248 p. 